\title{
Interprofessionalität schafft Syner- gien zum Nutzen der Patienten
}

\section{Michel Matter}

Dr., Mitglied des Zentralvorstands der FMH, Departementsverantwortlicher Dienstleistungen und Berufsentwicklung

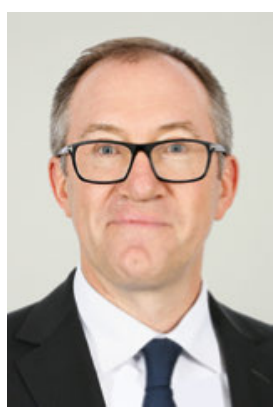

Die Zahl der Patienten, die an chronischen Erkrankungen leiden, nimmt in der Schweiz dramatisch zu. Das Schweizerische Gesundheitsobservatorium OBSAN schätzte 2015, dass diese Patientengruppe für fast 80 Prozent der Gesundheitskosten verantwortlich ist. Die Betreuung älterer und polymorbider Patienten die oftmals zu Hause erfolgt - ist komplex. Verschiedene Gesundheitsberufe setzen sich mit ihrer hohen fachlichen Kompetenz für die Patienten ein. Eine $\mathrm{Zu}$ sammenarbeit dieser Akteure schafft Synergien und ermöglicht eine deutliche Verbesserung der Versorgungsqualität.

An der Gründungsversammlung vom 22. Januar 2018 trat die FMH der "Plattform Interprofessionalität in der primären Gesundheitsversorgung» bei. Diese Plattform ist ein wichtiger Schritt nach vorne und ein starkes Signal, dass die verschiedenen Berufsverbände des Gesundheitswesens eine umfassende und qualitativ hochwertige interprofessionelle Versorgung fördern wollen. Das Interesse der Patienten und der Bevölkerung steht dabei im Vordergrund. Zusammenarbeit, Koordination, Management und Kompetenzentwicklung der einzelnen Organisationen werden in einer Einheit zusammengeführt. Dies gewährleistet eine aktive Vernetzung der verschiedenen Berufsgruppen. Mit diesem Beitritt bekräftigt die FMH ihre Bereitschaft, an diesem innovativen Ansatz für die primäre Gesundheitsversorgung mitzuwirken.

«Plattform Interprofessionalität in der primären Gesundheitsversorgung» - ein wichtiger Schritt nach vorne und ein starkes Signal.

Das Ziel dieser Plattform ist einerseits der Ideenaustausch, andererseits das Fördern eines besseren Verständnisses für die Arbeit der anderen Berufsgruppen. Um dies zu erreichen, müssen sowohl die Behandlungskoordination verbessert als auch wichtige Informationen sowie Daten bei der kontinuierlichen und integrierten Versorgung gemeinschaftlich genutzt werden. Dazu werden IT-Tools benötigt. In Genf beispielsweise konnten dank der Arbeit des Vereins PRISM der Plan zur gemeinschaftlichen Pflege (Shared Care Plan) und der Plan zur gemeinschaftlichen Medikation (Shared Medication Plan) eingeführt werden, inklusive Berücksichtigung der Interoperabilität und Gewährleistung des Datenschutzes. Die Barrieren bei der $\mathrm{Zu}-$ sammenarbeit zwischen den verschiedenen Gesundheitsberufen sollen Schritt für Schritt verschwinden und einer echten gemeinsamen Arbeit weichen, die auf neuen und verstärkten Synergien beruht. Dieses Netzwerk aus Kommunikation und Interaktion dient dazu, die Qualität der Versorgung zu verbessern. Selbstverständlich müssen die jeweiligen Kompetenzen der verschiedenen Akteure innerhalb eines definierten ethischen Rahmens geachtet und die Rolle jedes Akteurs sowie das grundlegende Verständnis der Verantwortlichkeit klar festgelegt werden.

\section{Innovative Projekte zur interprofessionellen} Zusammenarbeit sollen bereits im Rahmen der Ausbildung und Lehre unterstützt werden.

Auch der Patient muss seinen Teil zum Behandlungserfolg beitragen. Durch die Beteiligung der verschiedenen Akteure, die ihm mit professioneller Beratung zur Seite stehen, geschieht dies im Rahmen einer vertrauensvollen Beziehung. Zudem gilt es, innovative Projekte im Bereich der interprofessionellen Zusammenarbeit - auch bereits im Rahmen der Ausbildung und Lehre - umfassend zu unterstützen. Solche Projekte müssen weiterverfolgt und analysiert werden. Um die medizinischen, psychologischen und sozialen Bedürfnisse polymorbider Patienten zu befriedigen, ist ein interprofessionelles, patientenzentriertes Wissen in diesem Bereich von entscheidender Bedeutung.

Die interprofessionelle Arbeit erweist sich gerade jetzt als Chance, da die Kosten der Gesundheitsversorgung immer mehr im Mittelpunkt der Debatte stehen. Es braucht den starken Willen der Politik, ein Finanzierungsmodell zu finden, das Anreize für koordinierte Behandlungsteams setzt. Es gilt, die Fragmentierung der Behandlung zu bekämpfen, da sie hohe Kosten und eine verminderte Versorgungsqualität mit sich bringt. 